\title{
Spectral confocal reflection microscopy using a white light source
}

\author{
Martin J. Booth \\ martin.booth@eng.ox.ac.uk \\ Rimas Juškaitis
}

Tony Wilson

\author{
Department of Engineering Science, University of Oxford, Parks Road, Oxford, OX1 3PJ, United King- \\ dom \\ Department of Engineering Science, University of Oxford, Parks Road, Oxford, OX1 3PJ, United King- \\ dom \\ Department of Engineering Science, University of Oxford, Parks Road, Oxford, OX1 3PJ, United King- \\ dom
}

We present a reflection confocal microscope incorporating a white light supercontinuum source and spectral detection. The microscope provides images resolved spatially in three-dimensions, in addition to spectral resolution covering the wavelength range $450-650 \mathrm{~nm}$. Images and reflection spectra of artificial and natural specimens are presented, showing features that are not normally revealed in conventional microscopes or confocal microscopes using discrete-line lasers. The specimens include thin film structures on semiconductor chips, iridescent structures in Papilio blumei butterfly scales, nacre from abalone shells and opal gemstones. Quantitative size and refractive index measurements of transparent beads are derived from spectral interference bands. [D0I: 10.2971/jeos.2008.08026]

Keywords: confocal microscopy, supercontinuum, reflection, spectrometry

\section{INTRODUCTION}

The confocal microscope enables the imaging of volume specimens with three-dimensional resolution [1]. For biological imaging, it is most commonly used in fluorescence mode, in conjunction with appropriate fluorescent markers, to reveal structural and functional information about a specimen. The choice of illumination wavelengths had originally been restricted to the available laser lines, but the recent introduction of white light supercontinuum (WLS) sources has provided much greater freedom [2]-[4]. Whereas previously a confocal microscope could in practice use only a small number of laser wavelengths, a WLS source can now provide excitation at any wavelength in a range from around $400 \mathrm{~nm}$ to $2000 \mathrm{~nm}$. The combination of the WLS source with spectral detection has provided further versatility for confocal fluorescence microscopy $[5,6]$. The versatility of the WLS source in fluorescence microscopy has thus been widely demonstrated and well documented. However, it is also well suited for application to reflection mode confocal microscopy, such as the method already used to characterise the scattering from gold beads [7]. In this paper, we demonstrate the use of a WLS source in a reflection mode confocal microscope incorporating spectral detection. This microscope combines the three-dimensional spatial resolution of the confocal microscope with the spectral resolution of a spectrometer, providing image information that cannot be obtained using either conventional white light microscopy or confocal microscopy using discrete line laser sources. We present images and analyse spectra from a range of specimens that illustrate the capabilities of this method.

\section{MICROSCOPE IMPLEMENTATION}

The microscope configuration is illustrated in Figure 1(a). Broadband illumination (450-2000 $\mathrm{nm}$ ) was provided by a Fianium SC450-2 WLS source. The output light was passed through a filter, removing all wavelengths longer than $680 \mathrm{~nm}$ and the overall intensity was reduced by a factor of 1000 using a neutral density filter. The resulting beam was expanded then reflected by a $50: 50$ beam splitter into the back aperture of the objective lens (Olympus MPlanApo, 50×, 0.95 NA, dry). The specimen was mounted on a three-dimensional piezo translation stage (Physik Instrumente NanoCube). Light scattered by the specimen was collected using the same objective lens and was focused by a $200 \mathrm{~mm}$ focal length lens onto the $20 \mu \mathrm{m}$ diameter confocal pinhole. The light passing through the pinhole was recollimated by a $160 \mathrm{~mm}$ focal length lens before falling upon a blazed diffraction grating (600 line pairs $/ \mathrm{mm}$, blazed for $500 \mathrm{~nm}$ ) placed a distance of $210 \mathrm{~mm}$ away from the lens. The spectrally separated beam was passed via a scanning mirror and a $50 \mathrm{~mm}$ focal length lens onto the sensor chip of a CCD camera (Andor iXon EMCCD, $512 \times 512$ pixels, used in conventional detection mode). The distance between the grating and the mirror was $130 \mathrm{~mm}$ and that between the mirror and lens was $100 \mathrm{~mm}$. The resulting line focus on the CCD consisted of the reflection spectrum from a particular specimen point. As the specimen was scanned in the $x$ direction using a piezo translation stage, the galvanometric scan mirror would synchronously sweep the spectral line across the camera. This was achieved by driving both the stage and the mirror with the same scanning signal. By synchronising the camera exposure so that the shutter was open during a single scan, it was possible to retrieve a complete $x-\lambda$ "image" 

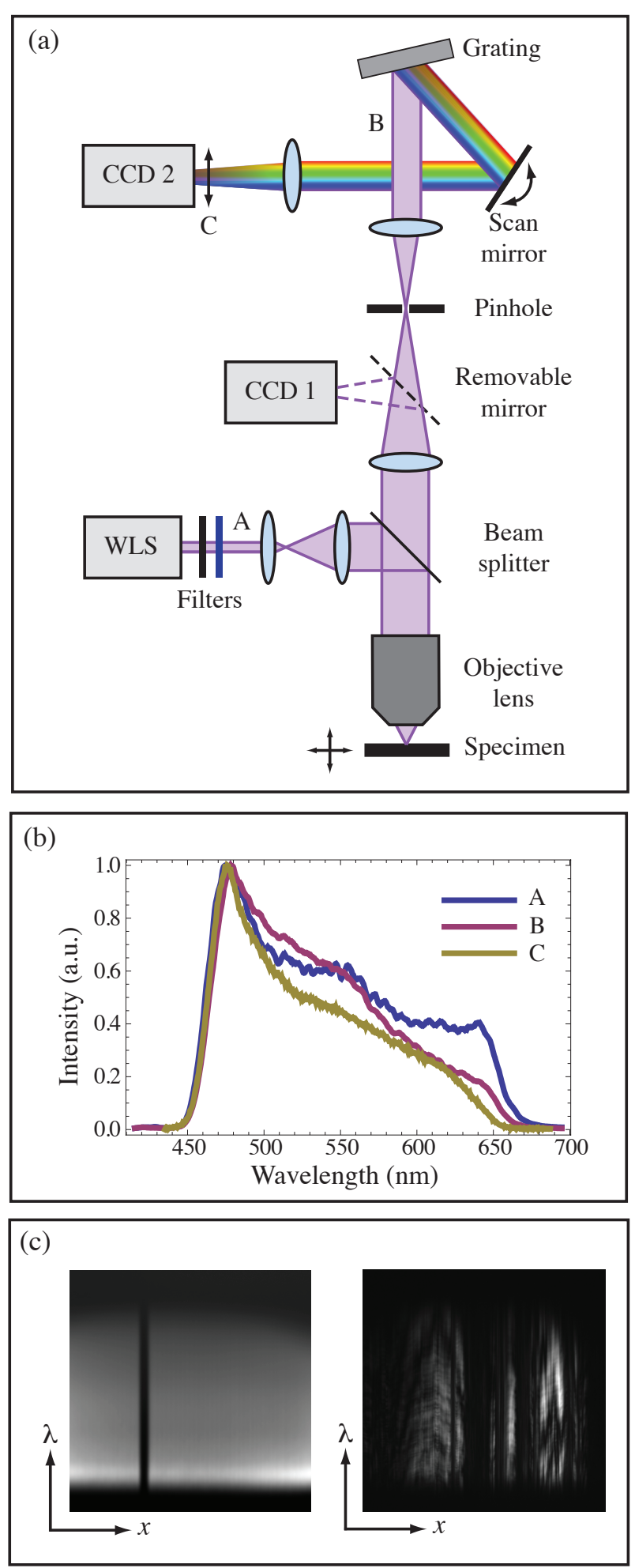

FIG. 1 (a) A schematic diagram of the spectral confocal reflection microscope. The the points denoted by letters $A, B$ and $C$ show the positions at which spectra were measured. WLS is the white light supercontinuum source. (b) The spectra at points A, $B$ and C. (c) Raw $x$ - $\lambda$ image frames from CCD 2. The image on the left is the reflection from a glass slide (the dark vertical stripe is due to an object on the slide obscuring the reflection). On the right is an example frame from the abalone specimen used in Figure 4 , showing detailed spectral and spatial structure. The $x$-axis image width is $100 \mu \mathrm{m}$.

in a single camera frame (Figure 1(c)). The microscope also included a second CCD camera, a removable mirror and conventional illumination to facilitate specimen location.

\section{SPECTRAL CALIBRATION AND PSEUDO REAL-COLOUR IMAGES}

In addition to spectral variations in the source and the wavelength dependent sensitivity of the CCD, various parts of the microscope could introduce chromatic effects, for example through absorption, reflection or diffraction. Figure 1(b) shows the spectra measured at three locations in the system. Spectrum A is the filtered output of the WLS at the input to the microscope, where the spectral variation is predominantly due to the source. Spectrum B was measured after the confocal pinhole, whilst using an aluminium mirror as the microscope specimen. This spectrum includes the effects of the losses in the objective lens and the dependence on wavelength of the power transmitted through the pinhole. Both A and B were measured using a calibrated Ocean Optics USB2000 spectrometer.

Spectrum C was measured using the CCD camera. The correspondence between wavelength and pixels was determined using the band edges produced by a set of bandpass filters at $483,518,568,628$, and $638 \mathrm{~nm}$. It was found that the spectrum was uniformly distributed across the pixels, such that the pixel pitch corresponded to a wavelength step of $0.50 \mathrm{~nm}$. As this relationship was linear, it was appropriate to consider the signal measured by the CCD pixels as a wavelength spectral density. In the results presented in this paper, the intensity spectra can therefore be regarded as equivalent to a spectral density (in arbitrary units).

For quantitative spectral reflectivity measurements, it was necessary to compensate for the non-uniform illumination spectrum, spectrally dependent losses in the optical system and detector sensitivity. For this reason, all measured pixel values, $p(\lambda)$, were normalised with reference to spectrum $C$, to give the renormalised reflection spectrum $r(\lambda)=p(\lambda) / c(\lambda)$. The calibration function $c(\lambda)$ was a smoothed version of spectrum $C$ obtained by taking a moving average of ten adjacent pixel values.

One method for visualising the spectral image data is to convert the data into pseudo real-colour red-green-blue (RGB) images. These images provide an approximation to the visual appearance of the genuine spectrum. Such images have been obtained before by using three discrete laser lines [8], but this method lacks the spectral coverage of the WLS source. We employed a simple method that is common to many colour imaging systems by summing particular ranges of the spectrum to generate each of the RGB values: the wavelength ranges from 450-500 nm, 500-580 $\mathrm{nm}$ and 580-650 nm were allocated to blue, green and red, respectively. In order to ensure an appropriate white balance, $x-z-\lambda$ data were taken of the reflection from the surface of a microscope slide. As there should be minimal spectral variation caused by this reflection, the infocus image should represent white and the relative proportions of the RGB components were adjusted to maintain this appearance. Using this calibration, the RGB images of other specimens were scaled accordingly. The $x-z-\lambda$ scan of the microscope slide also showed that the axial chromatic aberration was much smaller than the focal depth for this wavelength range. 


\section{RESULTS}

We investigated the capabilities of the white light confocal reflection microscope by imaging a semiconductor chip. Figure 2(a) shows a conventional microscope image of a region of the chip (obtained using a separate microscope), where the colours arise as a result of complex multilayered semiconductor structure and the varying thickness of the top passivation layer. The pseudo real-colour images in Figure 2(b) are from $x-z$ confocal sections through the surface of the chip at the various locations $\mathrm{A}$ to $\mathrm{E}$, demonstrating a range of colours. For all of these images, the illumination was incident from the top. Figure 2(c) shows the normalised spectra obtained at these points, showing significant spectral variation in the reflectance at the different points. The oscillating nature of the reflectance spectrum is typical of thin-film interference. The density plot of Figure 2(d) shows both the spatial and spectral variation in the region around point $\mathrm{D}$. The apparent shifting of the spectral bands could arise from small variations in the thickness of the transparent passivation layer.

The spectral confocal reflection microscope is particularly useful for imaging naturally occurring iridescent optical structures. Butterfly wings often have brightly coloured scales, whose optical properties arise from structures similar to photonic crystals $[9,10]$. Figure 3(a) shows $x-z$ sections of three different scales from a Papilio blumei butterfly (note that a gamma correction of 0.5 has been applied in order to enhance dim features). The confocal images reveal three-dimensional variations in reflectance of these scales. Note also the secondary foci produced by the curved surfaces of the concavities. Normalised reflection spectra from the points A to D are also presented. The different reflection spectra of the scales are clearly visible. These images are comparable with the optical and electron microscope images of Vukusic et al., who illustrate photonic structures in other Papilio species [10]. In particular, we note that the blue-biased spectrum D corresponds to finely structured ridges between the concavities. For all three scales, it can be seen that longer wavelengths appear to be reflected from a layer deeper than that which reflects the shorter wavelengths. This may be a consequence of a difference in optical properties of lower layers, as noted by Vukusic et al.

Iridescent structures are also produced in the shells of abalone [11]. Figure 4(a) shows pseudo real-colour $x-z$ and $x-y$ images of a fragment of abalone shell obtained using the spectral confocal microscope. The $x-y$ image was constructed as the maximum value pixel projection (sometimes referred to as an autofocus image) from four $x-y$ sections taken at $1 \mu \mathrm{m}$ axial steps. The striking colours are caused by the optical effects of stacks of aragonite platelets [11]. It is likely that the colour variations across the specimen are due to changes in the orientation of the stacks in different regions. For comparison, we also separately obtained conventional micrographs of an adjacent region of the specimen. The images were taken with a colour digital camera, using a 0.7 NA objective lens and halogen lamp illumination with both open and closed illumination aperture stop (Figure 4(a)). We note that the strong colour variation is not apparent in these images, as the cumulative effect of out-of-focus scatter leads to washed-out, predominantly white images. (a)

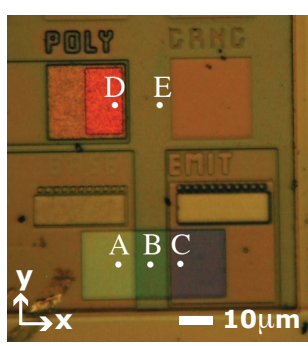

(b)

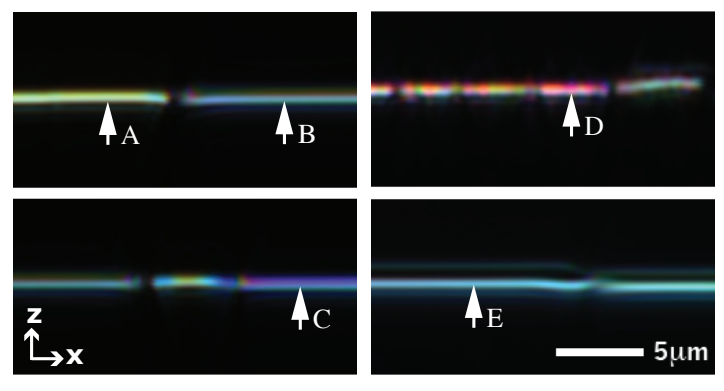

(c)
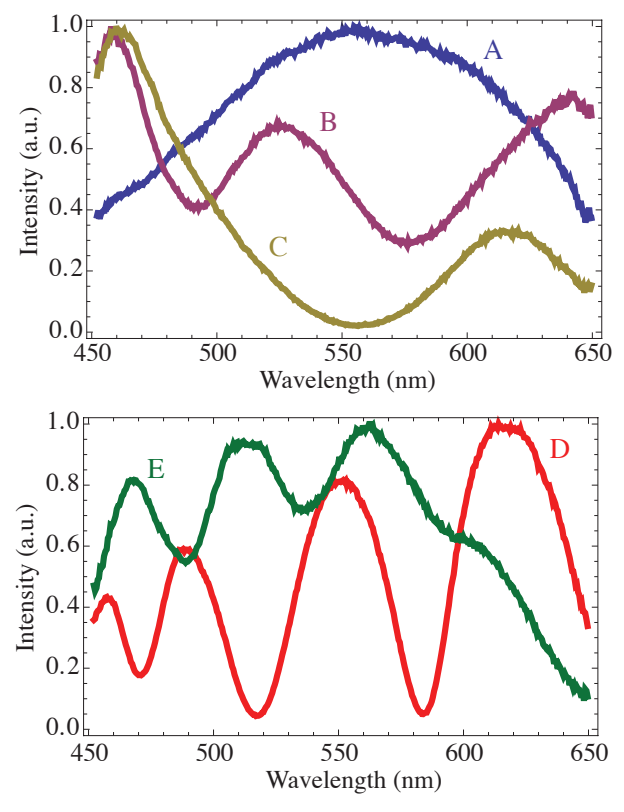

(d)

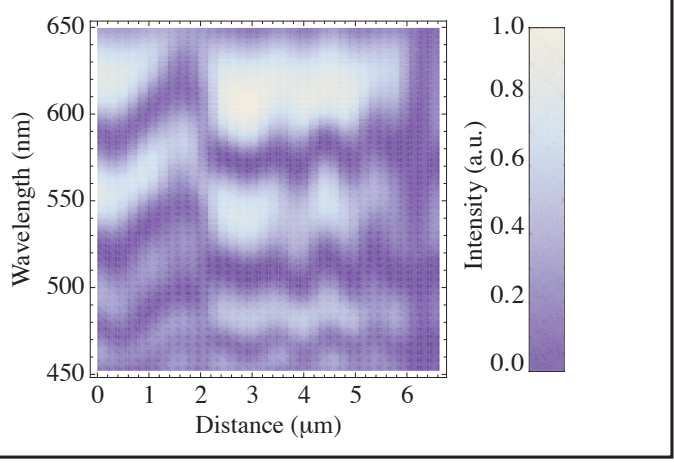

FIG. 2 Spectrally resolved images from an integrated circuit chip: (a) epi-illumination conventional microscope image of the chip showing approximate locations of the sample points A to E, taken using a 0.4 NA objective lens; (b) pseudo real colour $x-z$ images of parts of of the specimen; (c) normalised spectra measured at points $A$ to $E$; (d) $x-\lambda$ plot for a region centred on point $\mathrm{D}$. 


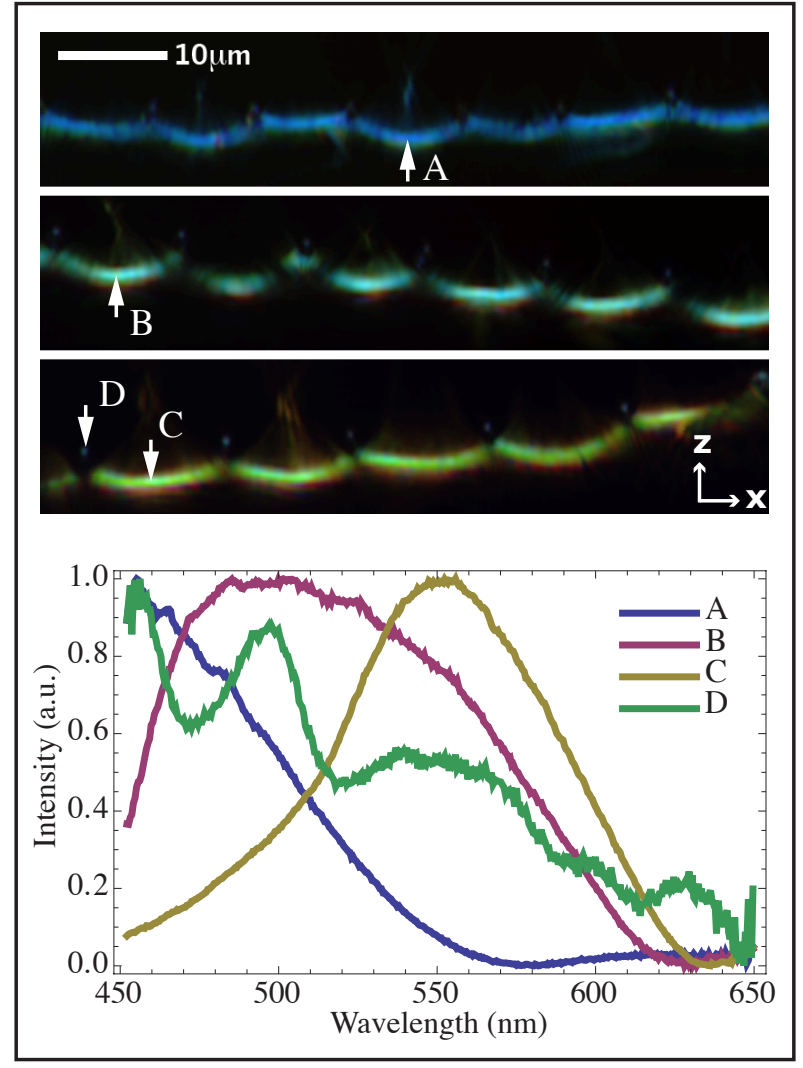

FIG. 3 Pseudo real colour $x-z$ confocal sections of three different scales from a Papilio blumei butterfly with spectra corresponding to the sample points A to D.

Figure 4(b) shows a single $x$-y image section of an opal gemstone. An opal consists of an array of amorphous silica spheres of uniform diameter, which typically lies in the range 150$400 \mathrm{~nm}$, depending upon the type of opal [12]. The spheres are locally arranged in a face centred cubic packing (occasionally hexagonal close packed), although there are domains over which the packing orientation changes. It is this structural variation that leads to the vivid iridescent colours of these gemstones. The resolution of the confocal microscope is insufficient to resolve the three-dimensional arrangement of the spheres. However, chromatic effects created by the structure, in particular bands of different colours, can be seen in Figure 4(b). Similar bands were observed by Akizuki [13], who suggested they were related to the change in orientation of the sphere packing in different domains. Again, it is important to note that this chromatic structure is not clearly revealed in high NA conventional microscope images of this specimen, which show a strong reflection from the surface (Figure 4(b)).

Many of the spectral features visible in these results are related to interference between reflections from different parts of the specimen. This effect is clearly seen in the refection spectra from transparent spherical beads, when the geometrical focus is coincident with the centre of the sphere (Figure 5). If we consider the geometrical optics approximation of a sphere that is much larger than the wavelength, all of the focused rays passing through the centre are normally incident on both the front and back surfaces of the sphere. The resulting normal reflections at each surface are similar in magnitude and follow a reciprocal path back through the objective lens. This results in a bright point in the confocal image, apparently from the centre
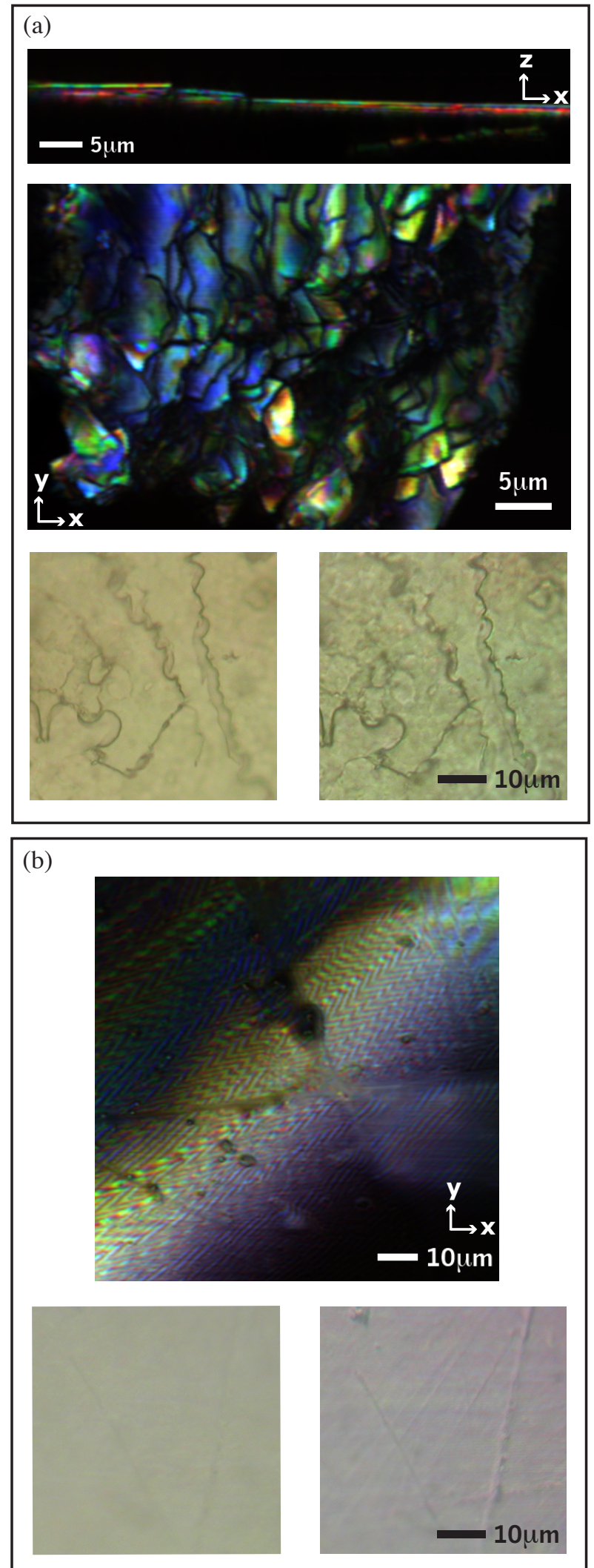

FIC. 4 Images from natural specimens: (a) pseudo-colour $x$-z confocal section (top), a maximum projection $x-y$ image (middle) and conventional images (bottom) of different locations in an abalone shell fragment. (b) $x$-y section of a natural opal gemstone (top) and conventional images of an adjacent region (bottom). The pairs of conventional images were obtained with the aperture stop open (left) and closed (right)

of the bead. By considering the interference between the external and internal surface reflections, one easily obtains the following expression relating the spectrum to the properties 

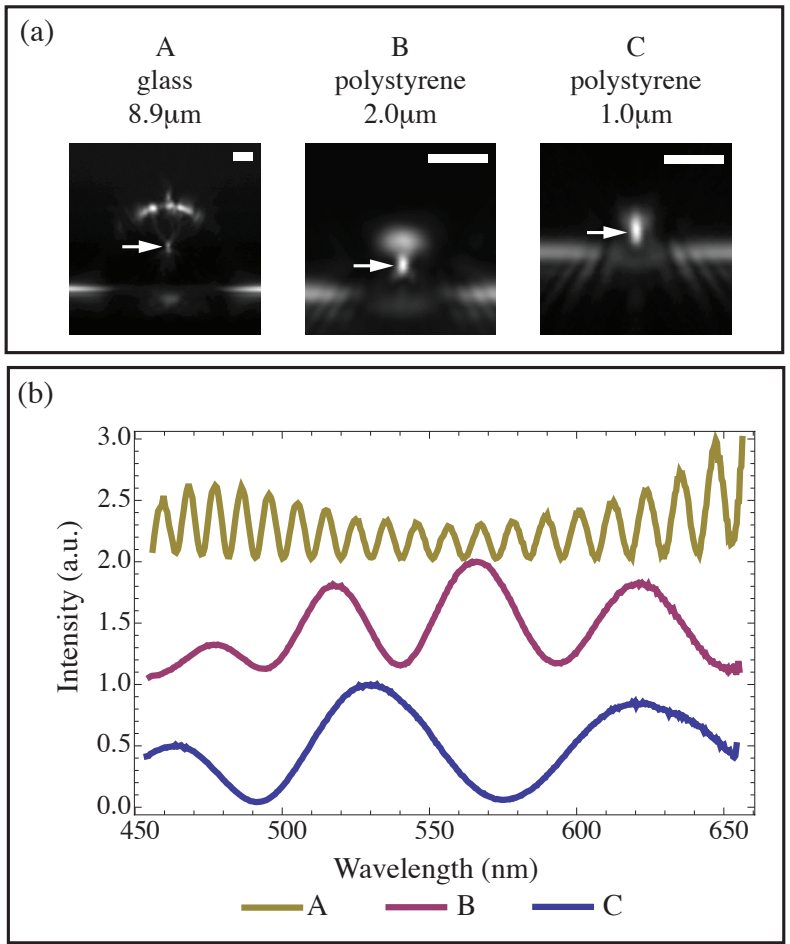

FIG. 5 Spectral confocal measurements of transparent beads: (a) Confocal $x$ - $z$ sections through the centres of three bead objects situated on a microscope slide. The greyscale pixel values represent total confocal reflection signal (a gamma correction of 0.67 has been applied to enhance the detail). The scale bars are $2 \mu \mathrm{m}$ long and the arrow indicates the point from which the spectra were obtained. (b) The corresponding reflection spectra obtained from the centre of each bead. For clarity, the curves for beads $A$ and $B$ have been offset along the vertical axis by 2 and 1, respectively.

of the bead:

$$
\frac{p}{2 n d}=\frac{1}{\lambda_{1}}-\frac{1}{\lambda_{2}},
$$

where $n$ is the refractive index of the sphere, $d$ is the diameter and $p$ is the number of spectral fringes between the wavelengths $\lambda_{1}$ and $\lambda_{2}$. Figure 5 shows $x-z$ confocal scans of three different beads placed on a microscope slide and the corresponding reflection spectra obtained from the apparent reflection at the centre of the bead. The glass beads were from Bangs Laboratories (IN, USA); the polystyrene beads were Microspheres from Molecular Probes, Invitrogen (CA, USA). For small beads (e.g. sample $C$ in Figure 5), the geometrical optics model of the reflections is not strictly valid. However, a strong signal with a similar spectral signature is still clearly present. From the spectra one can obtain estimates of the the refractive index or diameter of the beads. For specimen A the diameter was measured directly from the confocal microscope image as the height difference between the microscope slide and the top of the bead, giving $d=8.9 \mu \mathrm{m}$. From maxima in the spectrum we obtained $\lambda_{1}=462 \mathrm{~nm}$ and $\lambda_{2}=639 \mathrm{~nm}$ separated by $p=17$ fringes, giving the calculated refractive index as $n=1.59$. For the smaller polystyrene beads (B and $\mathrm{C}$ ), the diameters cannot be accurately determined from the confocal scans, but were specified by the manufacturers nominally to be $2.0 \mu \mathrm{m}$ and $1.0 \mu \mathrm{m}$ respectively. Using the value $n=1.59$ for the refractive index of polystyrene [14], we can estimate the actual diameter of the beads. For B we obtained from the two outermost maxima $\lambda_{1}=476 \mathrm{~nm}$ and $\lambda_{2}=621 \mathrm{~nm}$ separated by $p=3$ fringes, giving the calcu- lated diameter as $d=1.92 \mu \mathrm{m}$. For $\mathrm{C}$ we obtained from the two minima $\lambda_{1}=492 \mathrm{~nm}$ and $\lambda_{2}=573 \mathrm{~nm}$ separated by $p=1$ fringes, giving the calculated diameter as $d=1.09 \mu \mathrm{m}$. These values are close to the manufacturer's quoted nominal diameters.

\section{CONCLUSION}

We have shown that detailed spatial and spectral information can be obtained with a spectrally resolved reflection mode confocal microscope. This has enabled the imaging of specimen features that are not apparent in laser illuminated confocal microscopy or conventional microscopy. This microscope design could be further modified to use line, rather than spot, illumination and a slit instead of the pinhole in the detection path [15]. In this way, an entire $x-\lambda$ frame could be obtained in a single camera frame without the need for scanning in the $x$ direction, thus significantly improving the overal acquisition times of $x-y-z-\lambda$ data sets at the expense of slightly reduced axial resolution [16].

\section{ACKNOWLEDGEMENTS}

This work was supported by the Biotechnology and Biological Sciences Research Council. M. J. Booth was a Royal Academy of Engineering/EPSRC Research Fellow. The authors acknowledge the assistance of Dr. H. Townley with the butterfly specimens and Dr. T. Watanabe with the opal specimen.

\section{References}

[1] T. Wilson (ed.), Confocal Microscopy (Academic Press, London, 1990).

[2] C. Dunsby, P. M. P. Lanigan, J. McGinty, D. S. Elson, J. RequejoIsidro, I. Munro, N. Galletly, F. McCann, B. Treanor, B. Onfelt, D. M. Davis, M. A. A. Neil, and P. M. W. French, "An electronically tunable ultrafast laser source applied to fluorescence imaging and fluorescence lifetime imaging microscopy" J. Phys. D Appl. Phys. 37, 3296-3303 (2004).

[3] T. Betz, J. Teipel, D. Koch, W. Hartig, J. Guck, J. Kas, and H. Giessen, "Excitation beyond the monochromatic laser limit: simultaneous 3-D confocal and multiphoton microscopy with a tapered fiber as white-light laser source" J. Biomed. Opt. 10, 054009 (2005).

[4] G. McConnell, S. Poland, and J. M. Cirkin, "Fast wavelength multiplexing of a white-light supercontinuum using a digital micromirror device for improved three-dimensional fluorescence microscopy" Rev. Sci. Instrum. 77, 013702 (2006).

[5] J. H. Frank, A. D. Elder, J. Swartling, A. R. Venkitaraman, A. D. Jeyasekharan, and C. F. Kaminski, "A white light confocal microscope for spectrally resolved multidimensional imaging" J. Micros. 0xford 227, 203-215 (2007).

[6] D. M. Owen, E. Auksorius, H. B. Manning, C. B. Talbot, P. A. A. de Beule, C. Dunsby, M. A. A. Neil, and P. M. W. French, "Excitationresolved hyperspectral fluorescence lifetime imaging using a UVextended supercontinuum source" Opt. Lett. 32, 3408-3410 (2007).

[7] K. Lindfors, T. Kalkbrenner, P. Stoller, and V. Sandoghdar, “Detection and spectroscopy of gold nanoparticles using supercontin- 
uum white light confocal microscopy" Phys. Rev. Lett. 93, 037401 (2004).

[8] C. J. Cogswell, D. K. Hamilton, and C. J. R. Sheppard, "Color confocal reflection microscopy using red, green and blue lasers" J. Micros. Oxford 165, 103-117 (1992).

[9] H. Tada, S. E. Mann, I. N. Miaoulis, and P. Y. Wong, "Effects of a butterfly scale microstructure on the iridescent color observed at different angles" Appl. Opt. 37, 1579-1584 (1998).

[10] P. Vukusic, R. Sambles, C. Lawrence, and G. Wakely, "Sculptedmultilayer optical effects in two species of Papilio butterfly" Appl. Opt. 40, 1116-1125 (2001).

[11] T. L. Tan, D. Wong, and P. Lee, "Iridescence of a shell of mollusk Haliotis Glabra" Opt. Express 12, 4847-4854 (2004).
[12] J. V. Sanders, "Colour of precious Opal" Nature 204, 1151-1153 (1964).

[13] M. Akizuki, "Fractured surface of opal" Contr. Mineral. and Petrol. 28, 57-61 (1970)

[14] I. D. Nikolov, and C. D. Ivanov, "Optical plastic refractive measurements in the visible and the near-infrared regions" Appl. 0pt. 39, 2067-2070 (2000).

[15] R. A. Schultz, T. Nielsen, J. R. Zavaleta, R. Ruch, R. Wyatt, and H. R. Garner, "Hyperspectral imaging: A novel approach for microscopic analysis" Cytometry 43, 239-247 (2001).

[16] C. J. R. Sheppard, and X. Q. Mao, “Confocal microscopes With slit apertures" J. Mod. Opt. 35, 1169-1185 (1988). 